\title{
ZOOPLANCTON (CLADOCERA Y COPEPODA) DE LA LAGUNA DE CAIITTILĹN
}

\author{
Zooplankton (Cladocera and Copepoda) in Cajititlán Lagoon
}

\author{
Karl Edward Velázquez-Ornelas \\ Instituto de Ciencias del Mar y Limnología (ICMyL), Unidad Mazatlán, Universidad Nacional Autónoma \\ de México (UNAM), Av. Joel Montes Camarena, S/N CP 82040, Mazatlán, Sinaloa. \\ *Autor para correspondencia: kevelazquez@hotmail.com \\ Eduardo Juárez Carrillo \\ Instituto de Limnología (IL), Centro Universitario de Ciencias Biológicas y Agropecuarias (CUCBA). \\ Universidad de Guadalajara (UdeG). Paseo de la Loma 22, La Floresta, CP45920 Ajijic, Jalisco. \\ Manuel Ayón-Parente \\ Laboratorio de Ecosistemas Marinos y Acuicultura. Centro Universitario de Ciencias Biológicas y \\ Agropecuarias (CUCBA). Universidad de Guadalajara (UdeG). Camino Ramón Padilla Sánchez 2100 \\ Nextipac, CP45200 Zapopan, Jalisco.
}

\section{Resumen}

Los cladóceros y copépodos son los principales organismos formadores de zooplancton en aguas dulces. El estado de eutrofización y contaminación que enfrenta actualmente la laguna de Cajititlán restringe la cantidad de especies planctónicas que pueden habitar este cuerpo de agua, y con ello, la funcionalidad del ecosistema al ser estos los principales organismos consumidores de fitoplancton y la mayor fuente de alimento para peces y otros organismos. A pesar de la importancia que tienen ambos grupos para la fauna ictiológica, el conocimiento que se tenía sobre los ensamblajes de estos grupos en la laguna era escasa. Como resultado de este estudio se logró reconocer dos especies de cladóceros: Daphnia ambigua y Diaphanosoma birgei, y cuatro especies de copépodos: Arctodiaptomus dorsalis, Leptodiaptomus siciloides, Mesocyclops edax y Neoergasilus japonicus, de las cuales A. dorsalis y D. ambigua fueron las especies más abundantes y frecuentes. Neoergasilus japonicus se considera una especie invasora de origen asiático cuyas hembras adultas son parásitas de peces. La riqueza encontrada fue baja considerando el tamaño de la laguna, y las especies encontradas han sido asociadas con ambientes eutrofizados.

Palabras Clave: Cuerpos hídricos, especies exóticas, eutrofización.

\begin{abstract}
Cladocerans and copepods are the main zooplankton formers in freshwaters. The actual state of pollution faced by the lagoon of Cajititlán restricts the amount of species able to habit this water body, and with it, the functionality of the ecosystem due to their role as phytoplankton consumers and as the principal food source for fishes and other organisms. Until now, there was very little knowledge about these groups in the lagoon, which is because of the lack of specialists in the occidental region of Mexico and the inherent difficulties with respect to the manipulation of these organisms (their size round between 0.2 to $4 \mathrm{~mm}$, besides, manual dissections must be done in order to observe taxonomic characters). In this study, we analyze the composition and abundance of the zooplankton in Cajititlán Lagoon focused in both groups (Cladocera and Copepoda). To achieve this objective, five sites within the lagoon were chosen based on their position with respect to the principal human populations. Samples were collected using a plankton net of $63 \mu \mathrm{m}$ mesh light with one horizontal tow-in each site monthly. The analyzed period comprehends from May 2015 to April 2016, completing an annual cycle. Each sample was fixed using $4 \%$ formaldehyde. Organisms were first identified the species level and their abundance was calculated with manual counts using a Bogorov chamber. Six species were recorded: Daphnia ambigua and Diaphanosoma birgei (Cladocera) and Arctodiaptomus dorsalis, Leptodiaptomus siciloides, Mesocyclops edax and Neoergasilus japonicus (Copepoda). The most abundant and frequent species was A. dorsalis, followed by M. edax and D. ambigua. Leptodiaptomus siciloides and N. japonicus appeared to be rare species, but L. siciloides was very abundant in March and April. The most abundant species was A. dorsalis, occurring nearly in $77 \%$ of the samples and an average of 5 organisms per liter (up to 60 org/l), while the most frequent was M. edax, with up to $87 \%$ of occurrences and an average of 3 org/l (up to 80 org/l). The general abundance of both groups in this water body was below and the species composition corresponds to the expected in an eutrophicated waterbody.
\end{abstract}


Key words: Water bodies, exotic species, eutrophication

\section{Introducción}

El zooplancton es uno de los componentes bióticos principales en los cuerpos hídricos, por su importancia en las redes tróficas al ser los principales productores secundarios y sostener al resto de los consumidores (Elías-Gutiérrez y Sarma, 1999). La definición incluye organismos que se mantienen suspendidos en la columna de agua y que presentan una capacidad de desplazamiento relativamente limitada en comparación a organismos de mayor tamaño (Briseño-Avena, 2004). Por su tamaño tan reducido, se encuentran a merced de las corrientes generadas por el viento y los gradientes térmicos o de salinidad, y su distribución puede estar restringida a la zona fótica, mucho más productiva que el resto de la columna de agua (Symons et al., 2014).

En los cuerpos de agua dulce, el zooplancton es mucho menos diverso que en los océanos, aunque algunos grupos llegan a ser bastante abundantes y alcanzar densidades considerables. Los grupos que más contribuyen a la biomasa zooplanctónica en estos ecosistemas son: los cladóceros, los copépodos y los rotíferos, aunque pueden encontrarse también otros grupos, como los protozoos, invertebrados microscópicos (e.g., ácaros, tardígrados) larvas de peces y de crustáceos, nematodos y larvas de insectos (Rupert y Barnes, 1996; Ferdous y Muktadir, 2009; Elías-Gutiérrez, 2014).

En este artículo se abordará principalmente a los cladóceros y copépodos, que suelen constituir la parte más abundante del mesozooplancton de agua dulce y pueden llegar a alcanzar densidades que enturbian el agua si las condiciones lo permiten.

De acuerdo con Kotov et al. (2002), los cladóceros están representados a nivel mundial por aproximadamente 1,700 especies, incluyendo sinonimias. En cuanto a los copépodos, según Walther y Boxshall (2017), actualmente se han descrito unas 21,000 especies, que incluyen especies válidas, no aceptadas y sinonimias, aunque el número real varía alrededor de dieciséis mil especies, según otros autores.

La taxonomía de ambos grupos es un tema complicado, pues hay muchas discrepancias entre los autores, debido en buena parte a la dificultad de manipular y observar correctamente a los organismos por su reducido tamaño, que oscila en ambos grupos entre los 0.5 y los $3 \mathrm{~mm}$ en promedio. Además, pueden presentar una enorme variabilidad entre regiones, pues se ha observado que muchas especies son bastante plásticas, o que algunos taxa han resultado estar compuestos por especies crípticas (indistinguibles morfológicamente) (Elías-Gutiérrez et al., 2008a; Lee y Frost, 2002; Blanco-Bercial et al., 2014). También es común el determinar incorrectamente a los estadios de copepoditos como especies distintas, debido a su similitud considerable con los adultos, para lo que hay que tomar en cuenta el número de segmentos del urosoma, por ejemplo, el cual en los adultos consta de tres somitos, incluido el telson (Jaume et al., 2004).

Los cladóceros se encuentran representados en México por los órdenes: Ctenopoda (Sididae; Holopedidae), Anomopoda, (Bosminidae; Macrothricidae; Daphnidae; Moinidae; Ilocryptidae; y Chydoridae) y Onychopoda (Podonidae) (Elías-Gutiérrez et al., 2008b). El grupo cuenta con, al menos, 150 especies registradas hasta ahora en el país (ElíasGutiérrez y Sarma, 1999; Elías-Gutiérrez et al., 2008b).

Los copépodos, por otra parte, se encuentran representados (en agua dulce) por cuatro órdenes: Cyclopoida (Cyclopidae; Cyclopinidae; Lernaeidae), Calanoida (Diaptomidae; Pseudodiaptomidae; Centropagidae), Harpacticoida y Poecilostomatoida; sólo los primeros dos como verdaderos formadores de zooplancton (Suárez-Morales, 2000) y por aproximadamente 120 especies (Elías-Gutiérrez et al., 2008b).

Los números de taxones identificados para ambos grupos se encuentran en constante incremento. México cuenta con una diversidad alta de crustáceos, aunque las cifras registradas para muchos de los taxa son proporcionalmente bajas comparadas al resto del mundo (García-Madrigal et al., 2012).

\section{Características de los cladóceros}

El Orden que incluye a los cladóceros: Diplostraca, dentro de la Clase Branchiopoda, comprende representantes con morfologías muy variadas; todos presentan los pereiópodos en forma de hoja, mismos que les sirven como órganos para el intercambio gaseoso. Son generalmente filtradores o suspensívoros; presentan un caparazón formado por dos valvas que cubren el tórax y el abdomen, una cabeza que puede o no estar completamente fusionada al caparazón y seis pares de apéndices llamados toracópodos. Al igual que otros crustáceos, presentan dos pares de antenas: el primer par muy reducido en contraste con un segundo par bien desarrollado y con funciones locomotoras (Figura 1).

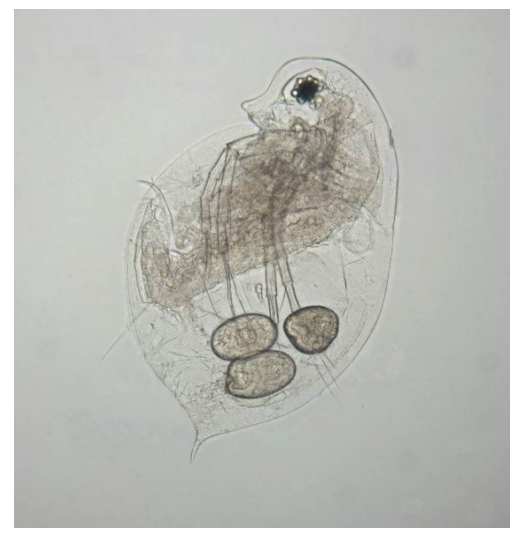

Figura 1. Hábito de una hembra ovígera de Daphnia ambigua en vista lateral Izquierda. Todos los cladóceros presentan un caparazón con forma de valvas que cubre por completo el tórax y abdomen.

Posterior a la región abdominal se ubica la sección que corresponde al telson y se denomina post abdomen, mismo que termina con un par de garras postabdominales, precedidas por una serie de espínulas o espinas en forma de 
peines o pectenes. Las pulgas de agua como comúnmente se les conoce, pueden presentar una coloración rojiza debido a su capacidad de sintetizar hemoglobina, misma que les sirve como mecanismo adaptativo a las concentraciones bajas de $\mathrm{O} 2$ disuelto en sus hábitats. La mayoría de los cladóceros son planctónicos, aunque existen especies con hábitos bentónicos. $\mathrm{Su}$ reproducción se da de manera asexual por vía partenogenética, generalmente. De manera adicional, aparecen machos cuando las condiciones son menos propicias y el intercambio de material genético es necesario. Para esto, las hembras desarrollan estructuras de resistencia conocidas como efipios, que constan de un cigoto recubierto por una membrana quitinosa e impermeable y que se encuentran unidos al exoesqueleto de la hembra. Su desarrollo es directo y los juveniles son versiones miniatura de los adultos (Rupert y Barnes, 1996).

\section{Características de los copépodos}

La Subclase copépoda, incluida dentro de la Clase Hexanauplia, puede distinguirse fácilmente por sus características morfológicas; presentan el primer par de antenas (anténulas) muy desarrolladas, mismas que tienen una gran variedad de funciones, desde locomotoras hasta reproductivas, mientras que las segundas antenas son generalmente muy reducidas y poco evidentes (Figura 2). Cuentan con entre 4 y 5 pares de pereiópodos que presentan estructuras acopladas a las coxas conocidas como escleritos intercoxales cuya función es la del batimiento sincrónico de las patas. El equivalente al primer par de patas se ha modificado como un par de maxilópodos. El cuerpo es cilíndrico o vermiforme y consta de un cefalosoma con 6 somitos y un tronco con 9 somitos más un télson, que es en realidad el conjunto del somito anal con un par de ramas caudales, también denominado furca. Los copépodos, a diferencia de los cladóceros, no tienen caparazón (Jaume et al., 2004).

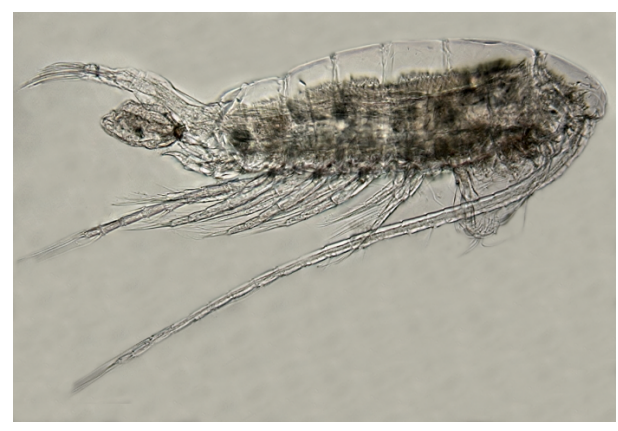

Figura 2. Hábito de una hembra de Arctodiaptomus dorsalis en vista lateral derecha. Los copépodos no tienen caparazón y su morfología recuerda generalmente a un grano de arroz diminuto.

Los copépodos presentan, de manera general, el quinto par de patas o pereiópodos reducidos o modificados con funciones prensiles (particularmente los machos) y en ocasiones este se encuentra reducido casi al grado de desaparecer por completo (Elías-Gutiérrez et al., 2008b). La forma de vida general del grupo es planctónica, aunque existen especies bentónicas o excavadoras, pueden existir incluso en películas de agua sobre la vegetación terrestre y sus hábitos alimenticios pueden ser filtradores, detritívoros, carnívoros o parásitos. Todos los copépodos presentan en su vida adulta un único ojo naupliar y aunque pueden parecer incoloros, generalmente tienen colores vivos o incluso bioluminiscencia. $\mathrm{Su}$ reproducción es típicamente sexual y suelen encontrarse proporciones similares de machos y hembras en la mayoría de las especies. Su desarrollo consiste en 6 etapas como nauplio y 5 como copepodito, que es una forma parecida al adulto, aunque sin segmentación definitiva (Rupert y Barnes, 1996).

\section{Antecedentes}

El estudio de los cladóceros en el país se ha concentrado principalmente en los estados del centro y sureste del país (Rodríguez-Almaráz y Leija-Tristán, 1995). La misma situación ocurre en el caso de los copépodos (Elías-Gutiérrez et al., 2008b).

Entre los trabajos realizados con los cladóceros continentales del país, se encuentran listados como aquel de Juday (1915) de la Ciudad de México; Wilson (1936) con especies de Yucatán; Úeno (1939) y Rioja (1940) para el Lago de Pátzcuaro; Osorio-Tafall (1943) los cladoceros de San Luis Potosí; van de Velde et al. (1978) un estudio para diversos estados del centro y sureste del país; RodríguezAlmaráz y Leija-Tristán (1995) del estado de Nuevo León, mientras que Ciros-Pérez y Elías-Gutiérrez (1996) proporcionan nuevos registros en México. trabajo de ElíasGutiérrez et al. (1999a) presenta los registros de cladóceros conocidos para todo el país.

Para el caso de los copépodos, algunos trabajos importantes para el país son aquellos de Fernando y Smith (1982), Collado et al. (1984), Reid (1990), y Fiers et al. (1996) con registros para América Central, México y el Caribe. Suárez-Morales y Reid (1996) realizaron un trabajo con muestras de Yucatán. Suárez-Morales y Reid (1998) efectuaron una actualización de los copépodos dulceacuícolas del país. Elías-Gutiérrez et al. (1999b) describen una nueva especie de Leptodiaptomus. Elías-Gutiérrez et al. (2008b) realizan una recopilación de los trabajos relacionados a la fauna de cladóceros y copépodos de México.

Los registros que se tienen de cladóceros y copépodos para el estado de Jalisco provienen de muestras del Lago de Chapala, algunas localidades del Río Lerma, de Chamela y, recientemente, de la Laguna de Cajititlán (Elías-Gutiérrez et al., 1999b; Elías-Gutiérrez et al., 2008b; Velázquez-Ornelas, 2017).

\section{Materiales y métodos}

Metodología para la identificación y análisis de datos Se analizaron muestras recolectadas mensualmente durante el ciclo anual mayo 2015 a abril 2016. Los muestreos se realizaron con una red cónica de $63 \mu \mathrm{m}$ de luz de malla y 30 cm de diámetro. 
Los arrastres fueron horizontales sobre la superficie con duración de un minuto, a una velocidad de un nudo (aproximadamente $2 \mathrm{~km} / \mathrm{h}$ ), con lo que se filtró un volumen final de aproximadamente $2.2 \mathrm{~m} 3 \mathrm{de}$ agua. Todas las muestras fueron fijadas in situ con formol al $4 \%$ y posteriormente preservadas con etanol al $70 \%$. En el caso de los cladóceros se revisaron únicamente hembras adultas ya que no se registró ningún espécimen macho. Los copépodos analizados corresponden a organismos de ambos sexos, también adultos. La identificación se realizó con base en caracteres sistemáticos morfológicos. Los ejemplares seleccionados fueron diseccionados y montados sobre un portaobjetos en una solución de glicerina (10\%) con etanol $(70 \%)$ y agua (20\%) para facilitar su manipulación y evitar su desecamiento y distorsión.

Las disecciones se realizaron con agujas de $0.2 \mathrm{~mm}$ de diámetro en un microscopio estereoscopio Carl Zeiss ${ }^{\circledR}$ Stemi 305 y la observación de los estados de carácter fue a través de un microscopio compuesto Carl Zeiss ${ }^{\circledR}$ Primo Star.

Los apéndices y estructuras con valor taxonómico usados en este trabajo son descritos a detalle por Elías-Gutiérrez et al. (2008b), Jaume et al. (2004) y Reid y Williamson (2010). La frecuencia de aparición de las diferentes especies se relacionó con la abundancia de cada una y se representó con un diagrama Olmstead-Tukey. Los datos de abundancia fuerontransformados a $\log (n+1)$ y analizados de acuerdo a Olmstead y Tukey (1974). En el diagrama de dispersión, dos líneas de referencia muestran la frecuencia relativa (eje X) y la abundancia mediana $(\log (n+1)$, eje $Y)$ de las especies de cladóceros y copépodos. El diagrama (Figura 3) muestra cuatro cuadrantes o esquinas en los que se representa a las especies dominantes, frecuentes, raras y abundantes.

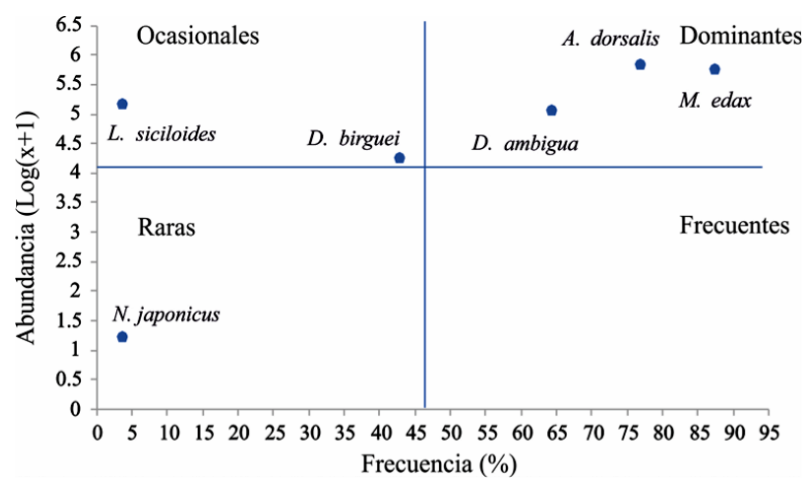

Figura 3. Diagrama de Olmstead-Tukey ilustrando la importancia biológica de cada una de las especies con base en su frecuencia y abundancia. Las líneas que separan los cuadrantes representan los promedios de cada vector.

Para cada especie registrada se presenta una diagnosis extendida con los principales caracteres morfológicos que permiten distinguirla. Asimismo, comentarios sobre su ecología y registro de distribución de las mismas.

\section{Resultados}

Del material examinado se registró la presencia de dos especies de cladóceros y cuatro de copépodos para la laguna. Asimismo, se registraron al menos tres morfo especies de rotíferos, larvas de dípteros y ostrácodos indeterminados taxonómicamente, en una proporción mucho menor. Los copépodos fueron siempre el componente dominante del zooplancton, alcanzaron hasta el $95 \%$ del total de organismos en las muestras (Figura 4).

La especie más abundante fue el copépodo Arctodiaptomus dorsalis, que apareció en $77 \%$ de las muestras con una densidad promedio de 5 organismos por litro filtrado, hasta un máximo de 60 org/l. Por otra parte, Mesocyclop edax fue la especie más frecuente, presente en un $87 \%$ de las muestras y con una abundancia media de 3 organismos por litro y hasta 80 org/l. Les siguió la especie Daphnia ambigua, con una frecuencia del $65 \%$ y abundancia promedio de 1.55 organismos por litro. Diaphanosoma birguei y Leptodiaptomus siciloides se comportaron como especies ocasionales (Figura 4); L. siciloides con abundancias de hasta 60 organismos por litro apareció únicamente en los meses de abril y mayo, mientras que D. birguei nunca superó los 2 organismos por litro.

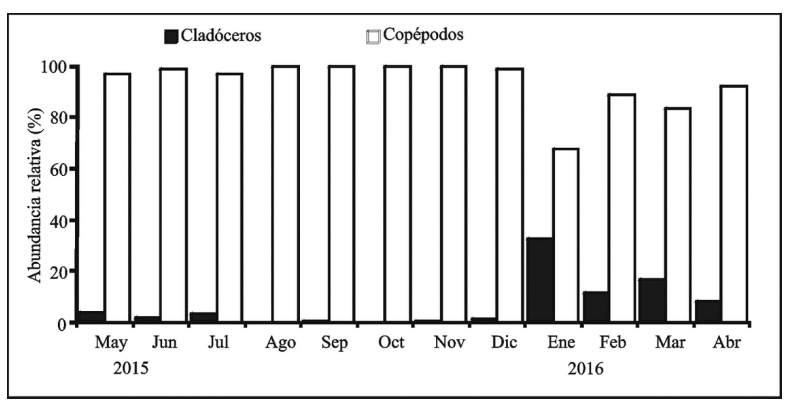

Figura 4. Abundancia relativa entre cladóceros y copépodos de la Laguna durante el ciclo de mayo 2015 a abril 2016.

Neoergasilus japonicus fue una especie rara, únicamente apareció en el mes de agosto, con apenas 50 organismos y la mayoría fijos a hospederos de los peces: Goodeaa atripinis, Gambusia sp. y Chapalicthys sp. Solo dos ejemplares juveniles de $N$. japonicus se registraron como parte del plancton. Uno de ellos se encontraba parasitando a un ejemplar de M. edax.

\section{Taxonomía de cladóceros y copépodos de la Laguna de Cajititlán}

Phyllum Arthropoda

Clase Branchiopoda

Orden Anomopoda

Familia Daphnidae

Género Daphnia

Daphnia ambigua Scourfield (1947) 
Al igual que otros dáfnidos, presenta un primer par de antenas inconspicuo, mucho más corto que el segundo par; cinco pares de apéndices torácicos diferenciables entre sí; efipios con uno o dos huevos. El caparazón (Figura 1) de esta especie presenta una espina terminal posterior, originada en la parte dorsal, de longitud menor a un tercio de la longitud del caparazón; el escudo cefálico penetra en el caparazón como una cuña en forma de " $V$ " en vista dorsal; es una especie pequeña, menor a $1 \mathrm{~mm}$, con las valvas circulares en vista lateral; margen interno de las valvas con pequeñas espínulas, presentes de la mitad hacia atrás.

En ocasiones forma un yelmo puntiagudo en la cabeza; entre los pectenes de la garra post abdominal no hay diferenciación evidente (Elías-Gutiérrez et al., 2008). Las anténulas de D. ambigua son relativamente pequeñas, su longitud no alcanza el margen distal del cuerpo; la base antenular es muy delgada en proporción al ancho de la cabeza al igual que otros dáfnidos. El material de Cajititlán representa un nuevo registro para el estado. Las especies de este género son particularmente resistentes ante condiciones de eutrofia debido a un sistema inmune bastante desarrollado y su capacidad de ingerir agentes patógenos sin recibir daños significativos (Ebert, 2005).

\section{Phyllum Arthropoda}

Clase Branchiopoda

Orden Ctenopoda

Familia Sididae

Género Diaphanosoma

Diaphanosoma birguei Korinek (1981)

Junto al resto de la familia Sididae, esta especie se caracteriza por tener seis pares de apéndices foliáceos, todos ellos similares entre sí; no forman verdaderos efipios y sus anténulas, aunque muy reducidas, tienen un segmento claramente diferenciado. Posee dentículos de diferente tamaño en el margen posterior de las valvas, formando grupos de dentículos pequeños intercalados entre los dentículos grandes; además, se observan setas intercaladas entre los grupos de dentículos pequeños y los dentículos grandes. Las anténulas (Figura 5) de D. birgei son relativamente grandes, su longitud alcanza el margen distal del cuerpo; la base tiene apariencia muy robusta, casi tan gruesa como el ancho de la cabeza, al igual que otros sídidos. Diaphanosoma birgei también representa un nuevo registro para Jalisco. Esta especie ha sido asociada con ecosistemas con presión predatoria (Doodson y Silva-Briano, 1996).

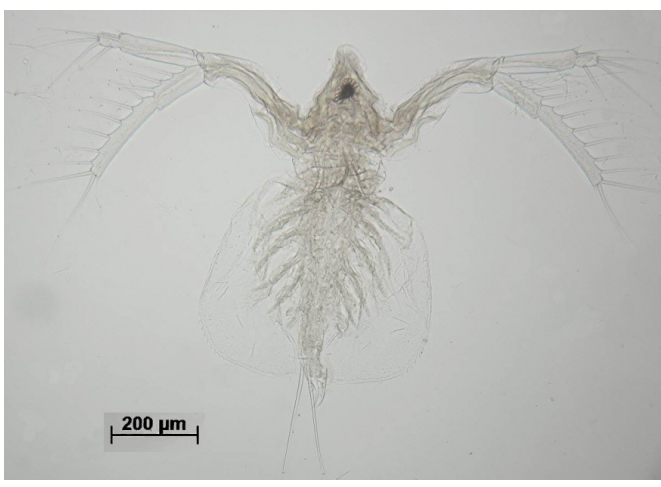

Figura 5. Diaphanosoma birguei, hembra en vista ventral. Las antenas de esta especie son mucho más grandes en proporción al cuerpo que las de un dáfnido.

Phyllum Arthropoda

Clase Hexanauplia

Orden Calanoida

Familia Diaptomidae

Género Arctodiaptomus

Arctodiaptomus dorsalis Marsh (1907)

Anténulas relativamente largas; sobrepasan el margen posterior de las setas caudales; proceso dorsal doble (Figura 6A) sobre el quinto segmento torácico en hembras (no todas las poblaciones de A. dorsalis presentan este carácter tan claramente (Reid, 2007)); anténulas con dos espinas en el segmento 11, anténula derecha del macho con procesos espiniformes en los segmentos 8, 10, 11 y 13, proceso en el antepenúltimo segmento de la anténula derecha del macho curvo y agudo, mismo cuya longitud es menor que la del penúltimo segmento, segmentos 14 y 15 de la anténula derecha del macho con procesos espiniformes fuertes. Cada rama caudal presenta 5 setas. Garra lateral externa del exópodo de la quinta pata derecha del macho (Figura 6B) se inserta en la mitad del segmento y es de mayor longitud que el mismo, proceso rectangular sobre el margen ventral del segundo segmento exopodal de la quinta pata derecha del macho (Elías-Gutiérrez et al., 2008b; Reid y Williamson, 2010; Haney et al., 2013). Es una especie de tamaño relativamente pequeño $(0.7-1.13 \mathrm{~cm})($ Reid, 2007).

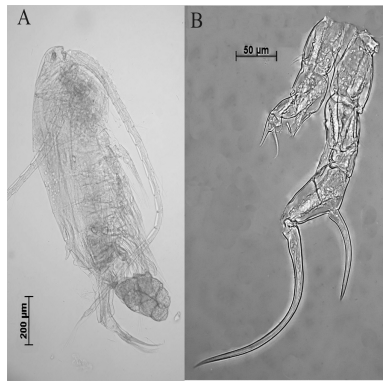

Figura 6. Arctodiaptomus dorsalis, A. hembra en vista lateral derecha. Nótese los procesos dorsales sobre los últimos dos segmentos del tórax. Las anténulas de esta especie son más largas que la longitud del cuerpo. B. quinto par de patas del macho en vista posterior. Presenta un proceso característico sobre el margen posterior del segundo segmento exopodal derecho y una garra lateral de casi la mitad de la longitud de la garra terminal. 
Es un taxón asociado a ambientes eutrofizados y con alta presión predatoria, con capacidad de resistir bastante bien el estrés ambiental. Su distribución es básicamente neotropical, aunque su origen se infiere en el paleártico (Reid, 2007).

Phyllum Arthropoda

Clase Hexanauplia

Orden Calanoida

Familia Diaptomidae

Género Leptodiaptomus

Leptodiaptomus siciloides Lilljeborj (1889)

Coxa de la quinta pata izquierda de la hembra (Figura 7A) con una espina grande en el margen externo; proceso rectangular hialino sobre el margen interno del primer segmento exopodal de la quinta pata derecha del macho; garra distal del exópodo derecho de la quinta pata con punta aguda (Figura 7B), dicha garra es más corta que el segmento (i.e., cerca de un tercio de su longitud). Espinas de los segmentos antenulares 8 y 10 de la anténula derecha del macho rectas, proceso del antepenúltimo segmento antenular de la anténula derecha del macho con el extremo distal redondeado. El metasoma en vista dorsal se ensancha en la parte media (ElíasGutiérrez et al., 2008b; Haney et al., 2013; Reid y Williamson, 2010). Es una especie que prefiere aguas cálidas, someras y turbias; su distribución es un potencial indicador de eutrofización, según Balcer et al. (1984). Su afinidad es principalmente paleártica. Representa el primer registro para el estado de Jalisco.

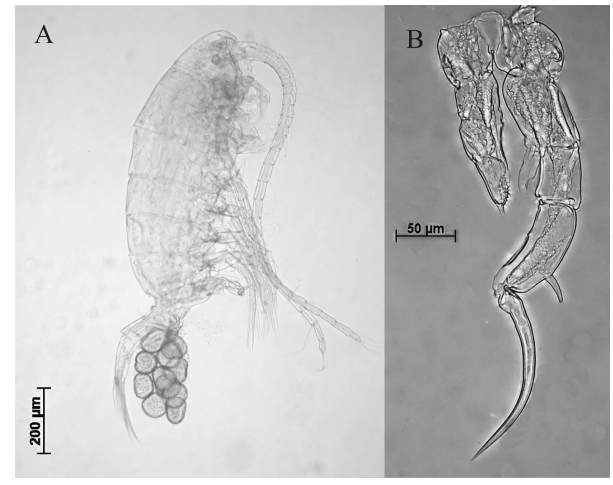

Figura 7. Leptodiaptomus siciloides. A. hembra en vista lateral derecha. En este caso, las anténulas son menos largas que el cuerpo. B. el quinto par de patas presenta una garra lateral recta mucho más corta que la garra terminal; además presenta un proceso característico de forma sub rectangular sobre el primer segmento exopodal de la pata derecha.

Phyllum Arthropoda

Clase Hexanauplia

Orden Cyclopoida

Familia Cyclopidae

Género Mesocyclops

Mesocyclops edax Forbes (1891)
Las quintas patas en $M$. edax son bisegmentadas, con dos setas largas de tamaño similar en el segmento distal y una seta espiniforme de longitud cercana a la mitad de las setas distales insertada en el segmento proximal; la seta interna del segmento distal se inserta en la sección media del segmento. Presenta anténulas de 17 segmentos. Los márgenes internos de las ramas caudales son pilosos (Figura 8). Al igual que otras especies del género, presenta una membrana hialina aserrada en los dos últimos segmentos de las anténulas en hembras con apariencia de sierra. El somito genital es cerca de dos veces más largo que ancho. (Elías-Gutiérrez et al., 2008b; Hudson et al., 2003).

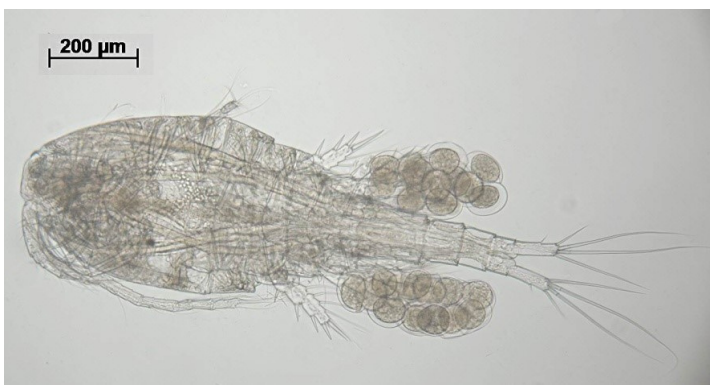

Figura 8. Mesocyclops edax, hembra en vista dorsal.

Mesocyclops edax es una especie que prefiere aguas cálidas. Su distribución es principalmente neártica. Sus hábitos alimenticios son de tipo predatorio, por lo que no depende del fitoplancton de manera tan directa como otros copépodos. (Elías-Gutiérrez et al., 2008b; Balcer et al., 1984). Este representa el primer registro en Jalisco.

Phyllum Arthropoda

Clase Hexanauplia

Orden Calanoida

Familia Ergasilidae

Género Neoergasilus

Neoergasilus japonicus Harada (1930)

Esta especie es de tamaño reducido, alrededor de $0.70 \mathrm{~cm}$ de longitud en las hembras; la forma del cuerpo es elongada (Figura 9). Presenta anténulas de 6 segmentos, de apariencia corta y robusta. Las antenas están modificadas para funciones prensiles, en forma de gancho (Figura 9B). 


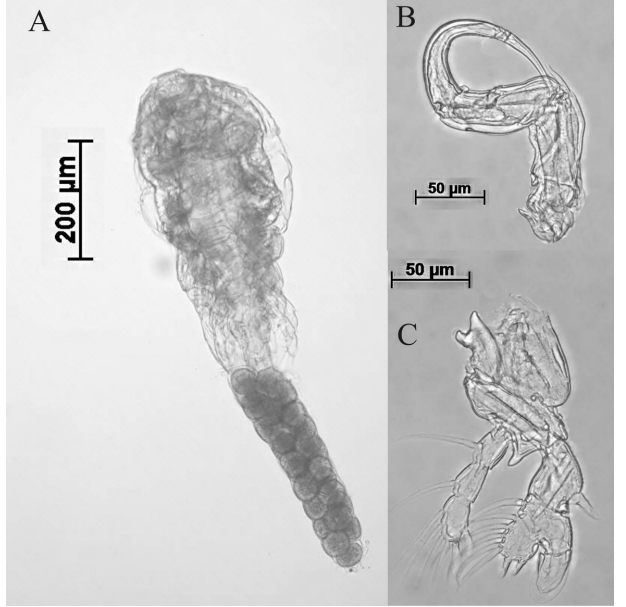

Figura 9. Neoergasilus japonicus. A. hembra (ovígera) en vista ventral. La longitud de los sacos ovígeros llega a ser igual o mayor que la del cuerpo del copépodo. B. antenas modificadas de la hembra, su principal función es el anclaje del copépodo al hospedero. La principal zona afectada son las aletas dorsal y anal. C. primera pata izquierda de la hembra. Nótese la espina en forma de pulgar sobre el segundo segmento del exópodo

El segundo segmento exopodal de la primera pata posee una espina lateral externa en forma de "pulgar", bastante robusta y redondeada, el cual es uno de sus atributos más característicos; además, en el primer segmento se observa una espina lateral externa muy desarrollada (Figura 10C). Las quintas patas tienen insertadas 3 setas en posición distal. Las cuartas patas en esta especie son unisegmentadas en ambas ramas. Las hembras ovígeras se distinguen fácilmente por la presencia de un par de sacos ovígeros que sobresalen desde el somito genital y llegan a ser tan largos como el cuerpo del organismo (Hayden y Rogers, 1998; Suárez-Morales et al., 2010).

Neoergasilus japonicus es una especie de origen AfroAsiático que parasita una gran variedad de especies de peces; hasta ahora se ha detectado su presencia sobre silúridos, centrárquidos, poecilidos y godeidos, entre otras familias (Suárez-Morales \& Mercado-Salas, 2013), e incluso se ha detectado parasitando a otros copépodos (Velázquez-Ornelas, 2017). Esta especie se incluye dentro de este trabajo debido a que tanto los machos como los juveniles forman parte del zooplancton, siendo de vida libre durante toda su vida; únicamente las hembras son parásitas, aunque pueden cambiar fácilmente de hospedero y tienen una inusual capacidad natatoria que no es común en otros ergasílidos (Hayden y Rogers, 1998). Su presencia en la laguna representa el tercer registro hasta ahora en México, después de haber sido detectado en Aguascalientes y en Chiapas. Su origen es paleártico; el primer registro proviene de Taiwán (Harada, 1930) y se sabe que se distribuye en gran parte de Europa y Norte América gracias a actividades relacionadas con la acuicultura (Suárez-Morales y Mercado-Salas, 2013).

\section{Conclusión}

La riqueza de cladóceros en la laguna está conformada por dos especies: Daphnia ambigua y Diaphanosoma birgei, mientras que la de copépodos consta de cuatro especies:

Daphnia ambigua y Diaphanosoma birgei, mientras que la de copépodos consta de cuatro especies: Arctodiaptomus dorsalis, Leptodiaptomus siciloides, Mesocyclops edax y Neoergasilus japonicus.

Todas las especies mencionadas en este trabajo, con excepción de A. dorsalis, son nuevos registros para Jalisco, con lo que se incrementa el listado de siete a nueve cladóceros y de cuatro a siete copépodos para el estado.

El grupo principal en cuanto a contribución de biomasa del zooplancton en la laguna son los copépodos.

Todas las especies registradas poseen adaptaciones que les permiten resistir, o incluso dominar, en ecosistemas eutrofizados, como esta laguna.

\section{Literatura citada}

- Balcer, M.D., Korda, N.L. y Dodson, S.I. (1984) Zooplankton of the great lakes: A guide to the identification and ecology of the common crustacean species. University of Wisconsin Press, Wisconsin, 174 pp.

- Blanco-Bercial L, Cornils, A. Copley, N. y Bucklin, A. (2014). DNA Barcoding of Marine Copepods: Assessment of Analytical Approaches to Species Identification. PLOS Currents Tree of Life. Edition 1. doi: $\quad$ 10.1371/currents.tol.cdf8b74881f87e3b01d 56b43791626d2.

- Briseño-Avena, C. (2004) Biomasa y composición del zooplancton de Bahía Chamela, Jalisco y Bahía Manzanillo, Colima durante un ciclo anual (2001-2002). Tesis de Licenciatura. Centro Universitario de Ciencias Biológicas y Agropecuarias, Universidad de Guadalajara, $58 \mathrm{pp}$.

- Ciros-Pérez, J. y Elías-Gutiérrez, M. (1996) Nuevos registros de Cladóceros (Crustacea: Anomopoda) en México. Biología Tropical, 44: 299-304.

- Collado, C., Fernando, C.H. y Sephton, D. (1984) The freshwater zooplankton of Central America and the Caribbean. Hydrobiologia, 113: 105-119.

- Doodson, S.I. y Silva-Briano, M. (1996) Crustacean zooplankton species richness and associations in reservoirs and ponds of Aguascalientes state, México, Hydrobiologia, 325: 163-172.

- Ebert, D. (2005). Ecology, Epidemiology, and Evolution of Parasitism in Daphnia. Bethesda (MD): National Library of Medicine (US), National Center for Biotechnology Information. Disponible de: http://www.ncbi.nlm.nih.gov/entrez/query.fcgi? $\mathrm{db}=$ Books

- Elías-Gutiérrez, M. (2014). Zooplancton de agua dulce: especies exóticas: posiblesvías de introducción. En: Mendoza Alfaro, R.E. y Koleff-Osorio, P. (Coords.) Especies acuáticas invasoras en México. Comisión 
nacional para el conocimiento y uso de la biodiversidad, México, pp. 309-340.

- Elías-Gutiérrez, M., Ciros-Pérez, J, Suárez-Morales, E., Silva-Briano, M. (1999a) Thefreshwater Cladocera (Orders Ctenopoda \& Anomopoda) of México, with comments on selected taxa. Crustaceana, 72: 171-186. http://www.ncbi.nlm.nih.gov/entrez/query.fcgi? $\mathrm{db}=$ Books (accesado el 31/12/17).

- Elías-Gutiérrez, M., Martínez-Jerónimo, F., Ivanova, N.V., Valdez-Moreno y Hebert, P.D.N. (2008a) DNA barcodes for Cladocera and Copepoda from Mexico and Guatemala, highlights and new discoveries. Zootaxa, 1839: 1-42.

- Elías-Gutiérrez, M. y Sarma, S.S.S. (1999) Zooplancton de sistemas acuáticos epicontinentales mexicanos en la región central de México. Universidad Nacional Autónoma de México, Facultad de Estudios Superiores Iztacala, Informe final SNIB-CONABIO proyecto No. H112, Ciudad de México, 197 pp.

- Elías-Gutiérrez, M., Suárez-Morales, E., GutiérrezAguirre, M.A., Silva-Briano, M. Granados-Ramírez, J.G. y Garfias-Espejo, T. (2008b) Cladocera y Copepoda de las aguas continentales de México, Comisión Nacional para el Conocimiento y Uso de la Biodiversidad, Facultad de Estudios Superiores-Iztacala (UNAM), El Colegio de la Frontera Sur, 322 pp.

- Elías-Gutiérrez, M., Suárez-Morales, E. y RomanoMárquez, B. (1999b) A new species of Leptodiaptomus (Copepoda, Diaptomidae) From Northwestern Mexico with comments on the distribution of the genus. Journal of Plankton Research, 21(4): 603-614.

- Ferdous, Z. y Muktadir, A.K.M. (2009) A review: Potentiality of zooplankton as bioindicator. American journal of applied sciences, 6 (10): 1815-1819.

- Fernando, C.H. y Smith, K.E. (1982) Copepoda. En: Hulbert, S.H. y Villalobos-Figueroa, A. (Eds.) Aquatic biota of México, Central America and West Indies. San Diego State University, San Diego, pp 192-195.

- Fiers, F., Reid, J.W. Iliffe, T.M. y Suárez-Morales, E. (1996). New hypogean cyclopoid copepods (Crustacea) from the Yucatan Peninsula, Mexico. Contributions to Zoology, 66 (2): 65-102.

- García-Madrigal, M.S., Villalobos-Hiriart, J. L., Álvarez, F. y Bastida-Zavala, R. (2012) Estado del conocimiento de los crustáceos de México. Ciencia y Mar. 16: 43-62.

- Haney, J.F. (2013). An-Image-based Key to the Zooplankton of North America" version 5.0. University of New Hampshire Center for Freshwater Biology, disponible desde: http://cfb.unh.edu/cfbkey/html/ (accesado el 29/12/2017).

- Hayden, K.J. y Rogers, W.A. (1998) Neoergasilusjaponicus (Poecilostomatoida: Ergasilidae), a Parasitic Copepod New to North America. The Journal of parasitology, 84: 88-93.
- Harada, I. (1930) Studies on the freshwater fauna of Formosa (I). A new copepod species parasitic on Formosan freshwater fish. Journal of Tropical Agriculture, 2: 71-76.

- Hudson, P.L., Lesko L.T., Reid, J.W. y Chriscinske, M.A. (2003) Cyclopoid copepods of the Laurentian Great Lakes. Ann Arbor, MI: Great Lakes Science Center Home Page. Disponible desde:http://www.glsc.usgs.gov/greatlakesco pepods/Key.php?GROUP=Cyclopoid (accesado el 01/01/2017).

- Juday, C. (1915) Limnological studies on some lakes in Central America. Wisconsin academy of sciences, arts and letters, 18: 214-250

- Jaume, D., Conradi, M. y López-González, P.J. (2004) Copépodos. En: Barrientos, J.A. (Ed.) Curso práctico de entomología. Universidad Autónoma de Barcelona, Barcelona, pp. 303-331.

- Juday, C. (1915) Limnological studies on some lakes in central America. Wisconsin academy of sciences, arts and letters, 18: 214-250

- Kotov, A., Forró, L., Korovchinsky, N.M. y Petrusek, A. (2002) World checklist of freshwater Cladocera species. Disponible desde: http://fada.biodiversity.be/CheckLists/CrustaceaCladocera.pdf (Último acceso 6 de enero de 2017).

- Lee, C.E. y Frost, B.W. (2002). Morphological stasis in the Eurytemoraaffinis species complex (Copepoda: Temoridae). Hydrobiología, 480: 111-128.

- Olmstead, P.S. y Tukey, J.W. (1974). A corner test for association. Annals Mathematical Statistics, 18 (4): 495513.

- Osorio-Tafall, B.F. (1943) Observaciones sobre la fauna acuática de las cuevas de la región de Valles, San Luis Potosí (México). Revista Sociedad Mexicana de Historia Natural, 4 (12): 47-51.

- Reid, J.W. (1990) Continental and coastal free-living Copepoda (Crustacea) of México, Central America and the Caribean region. Diversidad biológica de la reserva de Sian Ka'an, Quintana Roo, México. En: Navarro, D. y Robinosn J.G. (Eds.) Centro de investigaciones de Quintana Roo (CIQRO) and program of studies in tropical conservation. University of florida, Chetumal, Quintana Roo, México, pp. 175-213.

- Reid, J.W. (2007) Arctodiaptomusdorsalis (Marsh): A case history of copepod dispersal. Banisteria, 30: 3-18.

- Rioja, E. (1940) Observaciones acerca del plancton de Pátzcuaro. Anales del instituto de Biología, 11: 417-425.

- Rodríguez-Almaraz, G. y Leija-Tristán, A. (1995) Cladocerans (Branchiopoda: Anomopoda; Ctenopoda) ofthe Nuevo Leonstate, Mexico. Southwesthern Nature, 40:322-324.

- Ruppert, E. y Barnes, R. (1996). Zoología de los Invertebrados. Universidad de Complutense, Madrid, 
$1001 \mathrm{pp}$.

- Suárez-Morales, E. y Mercado-Salas, N. (2013) The nonindigenous parasitic copepod Neoergasilus japonicus (Harada) (Cyclopoida) from central Mexico: the earliest invasion in continental America. BioInvasors Records, 2: 201-206.

- Suárez-Morales, E. (2000). Copépodos, seres ubicuos y poco conocidos. CONABIO, Biodiversitas, 29: 7-11.

- Suárez-Morales, E., Paredes-Trujillo, A. y GonzálezSolís, D. (2010) The Introduced Asian Parasitic Copepod Neoergasilus japonicus (Harada) (Cyclopoida: Ergasilidae) from Endangered Cichlid Teleosts in Mexico. Zoological Science, 27: 851-855.

- Suárez-Morales, E. y Reid, J.W. (1996) Ampliación del ámbito de dos copépodos (Crustacea: Copepoda) de aguas continentales en la península de Yucatán, México. Revista de Biología Tropical, 44: 942-944.

- Suárez-Morales, E. y Reid, J.W. (1998) An updated checklist of the free-living freshwater copepods (Crustacea) of México. The southwestern naturalist, 43 (2): 256-265.

- Symons, C.C. Pedrusky, M.T., Arnott, S.E. y Sweetman, J.N. (2014). Spatial, Environmental, and Biotic Determinants of Zooplankton Community Composition in Subarctic Lakes and Ponds in Wapusk National Park, Canada. Artic, Antartic and Alpine Research, 46 (1): 159-190.

- Uéno, M. (1939) Zooplankton of Lago de Pátzcuaro, México. Otsu hydrobiological station, 18 (2): 105-115.

- Van de Velde, I., Dumont, H.J. y Grootaert, P. (1978) Report on a collection of Cladocera from Mexico and Guatemala. Archive furHydrobiologie, 83: 391-404.

- Velázquez-Ornelas, K.E. (2017) Composición y abundancia del zooplancton (Branchiopoda: Cladocera y Maxillopoda: Copepoda) durante 2015 y 2016 en la Laguna de Cajititlán, Jalisco, México. Tesis de Licenciatura, Universidad de Guadalajara, 49 pp.

- Walther, T.C. y Boxshall, G. (2017) World of Copepods data base. Disponible desde: http://www.marinespecies.org/copepoda (Último acceso 4 de enero del 2017).

- Wilson, C.B. (1936) Copepods from the cenotes and caves of Yucatán Peninsula, with notes on cladocerans. En: Pearse, A.S., Creaser, E.P. y Hall, F.G. (Eds.) The cenotes of Yucatán. 